\title{
UNIVERSAL EXPERIMENTAL RELATION FOR NATURAL FRIQUENCIES OF TRANSVERSAL VIBRATION OF STUBBY FREE-FREE BEAMS
}

\author{
V. A. Chirikov*, D. M. Dimitrov, K. P. Kostov \\ Technical University of Varna. Department of Technical Mechanics. 1 Studentska st., Varna, 9010, Bulgaria. \\ *Corresponding author. E-mail: chirikov@tu-varna.bg; address for correspondence: 1 Studentska st., Varna, 9010, Bul- \\ garia. Tel.: +359-52-383-686; fax: +359-52-302-771
}

On the basis of set of experimental tests a universal experimental relation for determination of natural transversal frequencies of short bulky bars with free ends is established. It is shown that the relation totally corresponds to approximate solution of the notorious differential equation obtained by Timoshenko for transversal vibration of stubby beams. The relation gives an opportunity for brief calculation of the transversal natural frequencies of stubby beams by avoiding complex and hard algorithms of vibration analysis. The achieved relation can be successfully used for control purposes of robot arms articulation, etc. An option of new differential equation for stubby both ends pinned beams transversal vibration is discussed.

Keywords: stubby beam, transversal vibration, natural frequency, universal relation.

DOI: $10.17804 / 2410-9908.2015 .4 .042-051$

\section{References}

1. Timoshenko S.P. On the Correction for Shear of the Differential Equation for Transverse Vibration of Prismatic Bars. Philosophical Magazine, 1921, vol.6, no. 41, pp. 744-746.

2. Timoshenko S.P. Kolebaniya $v$ inzhenernom dele [Vibration Problems in Engineering]. Moscow, Nauka Publ., 1967, 444 p. (in Russian).

3. Chirikov V.A. On the Range of Rotary Inertia and Shear Effects for Beams. In: Applied Mechanics in the Americas, PACAM VII. Temuco, Chile, 2002, vol. 9, pp. 9-12.

4. Chirikov Victor A., Koichi Ozaki. Experimental Formula for Transverse Vibrations of Stubby Free-Free Beams. In: International Conference "Tehnonav 2004”. Constantsa, Romania, 2004, pp. 25-28. ISSN 1223-7221.

5. Chirikov V.A. Uravneniya poperechnykh kolebaniy korotkikh balok [Equations for Transversal Vibrations of Stubby Beams]. Dep. in VINITI 06.12.2005, no. 1595-B2005. (In Russian).

6. Dimitrov D.M. Application of dynamic methods for the determination of elastic constants of materials produced by powder metallurgy. Nauchni Izvestiya na NTSM, june 2013, vol. XXI, iss. 2(139), pp. 433-435. ISSN 1310-3946. (In Bulgarian).

7. Kostov K. Determination of natural frequencies of beams with distributed parameters for free vibration. In: Yubileina studentska nauchna sessiya na TU-Varna, 04.04.2012. (In Bulgarian). 8. ASTM E1876-09 Standard Test Method for Dynamic Young's Modulus, Shear Modulus, and Poisson's Ratio by Impulse Excitation of Vibration. Book of Standards Volume: 03.01. ASTM, USA, 2012. 
Подана в журнал 20.08.2015

УДК 534-16.011

DOI: $10.17804 / 2410-9908.2015 .4 .042-051$

\title{
УНИВЕРСАЛЬНАЯ ЭКСПЕРИМЕНТАЛЬНАЯ ЗАВИСИМОСТЬ ДЛЯ ОПРЕДЕЛЕНИЯ СОБСТВЕННЫХ ЧАСТОТ ПОПЕРЕЧНЫХ КОЛЕБАНИЙ КОРОТКИХ БАЛОК СО СВО- БОДНЫМИ КОНЦАМИ
}

\author{
В. А. Чириков*, Д. М. Димитров, К. П. Костов \\ Кафедра Технической механики, Технический университет - Варна, ул. Студентска 1, \\ 2. Варна 9010, Болгария.
}

*Ответственный автор. Электронная почта: chirikov@tu-varna.bg; адрес для переписки: ул. Студентска 1, г. Варна 9010, Болгария. Телефон: +359-52-383-686; факс: +359-52-302-771.

На основании серии экспериментальных испытаний установлена универсальная экспериментальная зависимость для определения собственных поперечных частот коротких цельных стержней со свободными концами. Продемонстрировано, что это соотношение полностью соответствует приближённому решению известного дифференциального уравнения для поперечных колебаний коротких балок, полученного Тимошенко. Установленная зависимость даёт возможность лаконично, без сложных и трудоемких алгоритмов анализа колебаний, рассчитать поперечные собственные частоты коротких балок. Полученную зависимость можно с успехом применять в целях управления шарнирными соединениями рук робота и т. п. В качестве варианта рассмотрено новое дифференциальное уравнение для поперечных колебаний коротких балок с обоими закреплёнными концами.

Ключевые слова: короткая балка, поперечные колебания, собственная частота, универсальная зависимость.

\section{1. Введение}

При моделировании колебаний подавляющего числа элементов конструкций, строений, звеньев машин, современных роботов-манипуляторов и пр., применяемая классическая теория Эйлера-Бернулли, которая пренебрегает эффектами инерции вращения и сдвига поперечных сечений, вносит серьезную погрешность при проектировании и управлении таких объектов.

Примером сказанному могут послужить манипуляторы современных промышленых роботов, звенья которых часто представляют короткие массивные тела, значительно болеее тяжелые, чем грузы, для транспортировки которых они предназначены. Отношение массы всего манипулятора к массе груза обычно варьируется в рамках от 30 до 50. Причиной этому является необходимость получения нужной жесткости звеньев с целью обеспечения точности выполнения рабочих операций. Поэтому при динамическом моделировании звеньев промышленых манипуляторов необходимо иметь в виду, что они представляют собой короткие массивные тела, которые в свою очередь моделируются как короткие балки. При колебаниях таких объектов следует учитывать эффекты инерции вращения и сдвига чтобы избежать серьезной погрешности, особенно в отношении адекватности алгоритмов их управления.

Впрочем, учет упомянутых эффектов приводит к возникновению других серьезных трудностей. К ним относится необходимость примения более сложных алгоритмов управления, которые затрудняют работу манипуляторов и уменьшают эффективность их применения. Очевидна необходимость дальнейшего усовершенствования приближенных методов расчета поперечных колебаний коротких массивных тел, включая короткие балки, 
которые смогли бы упростить алгоритмы управления и привести к более точному моделированию реальных процессов колебаний.

В настоящем исследовании проведена экспериментальная проверка реального процесса поперечных колебаний серии массивнных коротких балок со свободными концами и установлена универсальная зависимость, удобная как для экспрессных расчетов частот таких колебаний, так и для использования в эффективных алгоритмах управления. Проведен анализ полученной зависимости на соответствие с теорией Тимошенко [1] поперечных колебаний коротких балок с учетом инерции вращения и сдвига, который подтвердил, уточнил и теоретически обосновал полученный результат. В работе обсуждается возможность применения полученной зависимости для поперечных колебаний коротких балок с другими краевыми условиями.

\section{2. Экспериментальная постановка}

Для исследвания изготовлена специальная серия из 19 коротких стальных балок различной длины с плотными квадратными поперечными сечениями. Стороны квадратов имеют приблизительные размеры 4,5 cм, а длины балок находятся в диапазоне приблизительно от 11,5 до 30 см. Размеры поперечных сечений измерялись с точностью до 0,01 мм, а длины балок с точностью до 0,1 мм. Результаты каждой серии замеров отдельных балок осреднялись. Подробные геометрические размеры балок даны в табл. 1.

Таблица 1. Геометрические характеристики образцов балок

\begin{tabular}{|c|c|c|c|}
\hline$№$ & Длина $-\mathrm{L}, \mathrm{M}$ & Ширина $-\mathrm{b}, \mathrm{m}$ & Высота $-\mathrm{h}, \mathrm{m}$ \\
\hline 1 & 0,115 & 0,045 & 0,04535 \\
\hline 2 & 0,12 & 0,04495 & 0,0452 \\
\hline 3 & 0,1236 & 0,045 & 0,04525 \\
\hline 4 & 0,13 & 0,045 & 0,04525 \\
\hline 5 & 0,134 & 0,04495 & 0,0453 \\
\hline 6 & 0,14 & 0,0449 & 0,04515 \\
\hline 7 & 0,144 & 0,04495 & 0,0452 \\
\hline 8 & 0,15 & 0,045 & 0,0452 \\
\hline 9 & 0,16 & 0,0451 & 0,04525 \\
\hline 10 & 0,17 & 0,04505 & 0,04525 \\
\hline 11 & 0,18 & 0,04495 & 0,04525 \\
\hline 12 & 0,189 & 0,0451 & 0,04525 \\
\hline 13 & 0,199 & 0,04525 & 0,04525 \\
\hline 14 & 0,209 & 0,04495 & 0,04525 \\
\hline 15 & 0,229 & 0,0449 & 0,0452 \\
\hline 16 & 0,249 & 0,04495 & 0,0452 \\
\hline 17 & 0,26 & 0,04495 & 0,04515 \\
\hline 18 & 0,281 & 0,0449 & 0,0451 \\
\hline 19 & 0,302 & 0,04485 & 0,0451 \\
\hline
\end{tabular}

Материал всех образцов - сталь 45 по БДС EN10060, для которой установлены следующие средние значения [6]: массовая плотность материала $\rho=7699 \kappa 2 / \mathrm{m}^{3}$, модуль упругости $E=205,7$ ГПа и коэффициент Пуассона $v=0,25$. При этом вес образцов измерялся с точностью до 1 грамма. Плотность получена как отношение веса к объему. Модуль упругости получен по методике ASTM E1876-09 [8]. При расчетах принималось указанное выше приблизительное значение коэффициента Пуассона. Для измерения 
open-acCeEs journal

основных собственных частот колебаний отдельных образцов балок использовалась постановка эксперимента, представленная на рис.1.

Исследуемая балка устанавливалась на массивном фундаменте, содержащем две угловые опоры (неподвижную и подвижную), обеспечивающие свободное колебание балки в вертикальной плоскости (см. рис. 1).

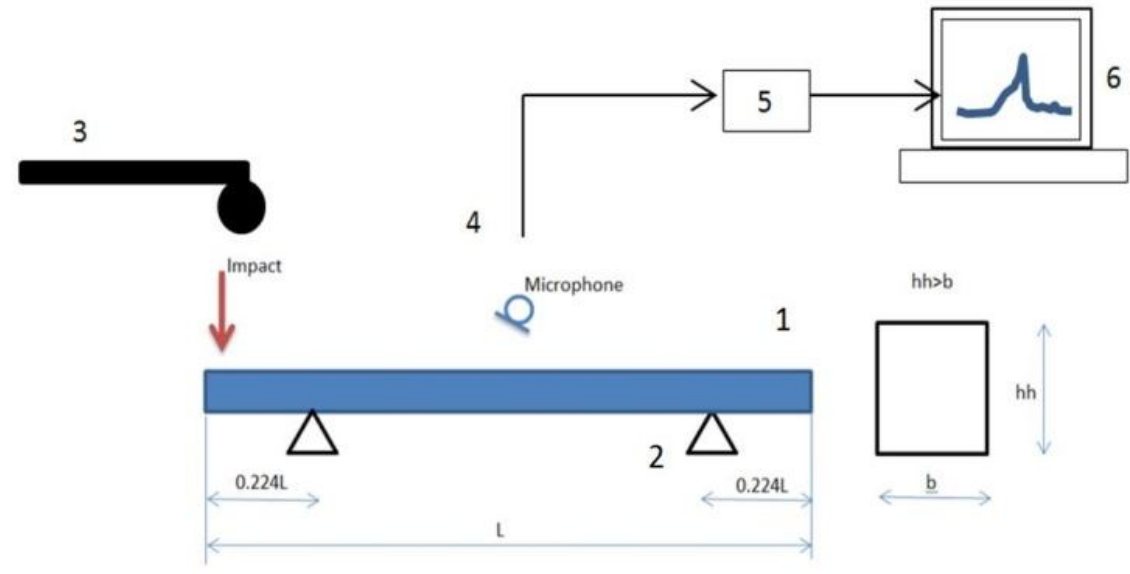

Рис.1 Схема эспериментальной постановки:

1 - образец; 2 - опоры; 3 - импульсный молоток; 4 - микрофон;

5 - цифровой осциллоскоп; 6 - компьютер

Балка (1) устанавливалась на опорах таким образом, чтобы больший из двух измеренных размеров поперечного сечения балки являлся высотой, а контактные линии опор находились в характерных узловых точках соответствующей балки (2). При помощи импульсного молотка (3), который представляет собой шарик из закаленной стали, прикрепленный к гибкой рукоятке, производится легкий удар по концу балки. Вибрации регистрируются посредством микрофона МК122 (4), чей сигнал подается на цифровой осциллоскоп Picoscope 2206 (5). Инсталлированное на компьютре (6) программное обеспечение осциллоскопа преобразует поступающий сигнал в частотный спектр, откуда определяется соответствующая основная частота колебаний балки. Для контроля получаемых результатов собственная частота балок определяется путем расчета по классической формуле Бернулли и при помощи программного пакета Comsol Multiphysic 4.3b (Structural mechanic module, Beam, Eigenfrequency analysis) [7].

Результаты экспериментов и параллельного счета для каждой балки представлены на следующем рис. 2.

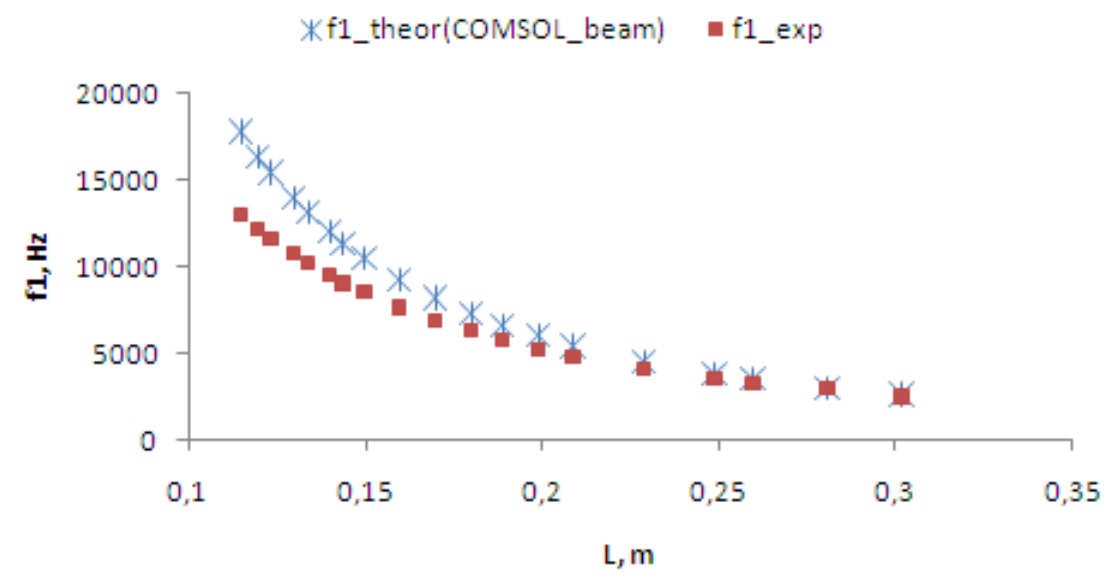

Рис. 2. Частоты поперечных колебаний коротких балок со свободными концами:

*- расчетное значение классической частоты; - - экспериментальное значение частоты

Chirikov V. A. et al. / Universal experimental relation for natural friquencies of transversal vibration of stubby

free-free beams 
Очевидно, что при более длинных балках классические и экспериментальные значения частот совпадают, а чем короче балки, тем различие становится сильнее. Это объясняется увеличением эффектов инерции вращения и сдвига поперечных сечений балок. При этом экспериментальные данные показывают, что эти эффекты приводят ко все большему уменьшению собственных частот поперечных колебаний коротких балок со свободными концами. Кроме того очевидно, что экспериментальные данные ложатся на некую кривую, вид которой устанавливается ниже.

\section{3. Универсальная зависимость для расчета частот поперечных колебаний коротких ба- лок со свободными концами}

Кроме полученных результатов, представленных на рис. 2, для удобства анализа предлагается использовать специальную координатную систему с параметром $s$ по абсциссе и собственной частотой $\omega_{n}$ по ординате [3]. Безразмерный параметр $s$ включает в себя геометрические и инерционные характеристики балок, виды опор и номер собственной частоты, и имеет вид:

$$
s=\frac{L}{n_{c} r},
$$

где: $L$ - длина балки; $r$ - инерционный радиус поперечного сечения, а $n_{c}$ - частотное число, получаемое из решения уравнения детерминанты формы колебаний балки.

Например, для балок на шарнирных опорах по концам, последовательные частотные числа $n_{c}$ равняются порядковому номеру частоты $n$, т.е. $n_{c}=n$.

Для жестко закрепленных с обеих сторон балок или балок со свободными концами последовательные частотные числа являются следующими: 1,$505619 ; 2,499753 ; 3,50001 ; 4,5$ и т.д. Для балок с одним шарнирным концом, а другим жестко закрепленным или свободным: 1,$249876 ; 2,249999 ; 3,25 ; 4,25$ и т.д. Для консоли они следующие: 0,5996864; 1,$494176 ; 2,500247 ; 3,5 ; 4,5$ и т.д.

Общий член этих рядов для высших частот можно легко определить. Для жестко закрепленных с обеих сторон балок или балок со свободными концами: $n_{c}=n+0,5$. Для балок с одним закрепленным шарнирно концом, а другим жестко закрепленным или свободным: $n_{c}=n+0,25$. Для консоли: $n_{c}=n-0,5$. Очевидно, что нулевые значения частотных чисел, относящихся к движениям твердого тела, в эти ряды не включаются. Для таких значений параметр $s$ становится бесконечно большим.

По оси ординат откладываются собственные частоты колебаний балок $\omega_{n}$. Благодаря введенному параметру $s$, любую классическую частоту поперечных колебаний длинной тонкой балки с типичными краевыми условиями на концах можно представить следующим образом:

$$
\omega_{n}=\frac{c}{r}\left(\frac{\pi}{s}\right)^{2}
$$

где вводится обозначение $c=\sqrt{\frac{E}{\rho}}$.

Получается уравнение квадратной гиперболы. Для удобства представления результатов дополнительно введем следующий безразмерный параметр $z$ : 
opent-aceess jurnal

$$
z=\left(\frac{\pi}{s}\right)^{2} .
$$

С его помощью собственные частоты $\omega_{n}$ колебаний длинных балок можно представить следующей зависимостью:

$$
\omega_{n}=\frac{c}{r} \cdot z
$$

При настояшем исследовании получаемые значения собственных частот поперечных колебаний коротких балок со свободными концами сравнивались с аналитической зависимостью:

$$
\omega_{n}=\frac{c}{r} \cdot \frac{z}{\sqrt{1+4 z}}
$$

установленной при проведении экспериментов в Токайском университете, Япония [4]. Различие с настоящей постановкой эксперимента состоит в том, что исследуемые тогда балки подвешивались вертикально на нерастяжимой нити.

Сравнение пересчитанных значений результатов тестов с предлагаемой зависимостью представлено на следующем рис. 3.

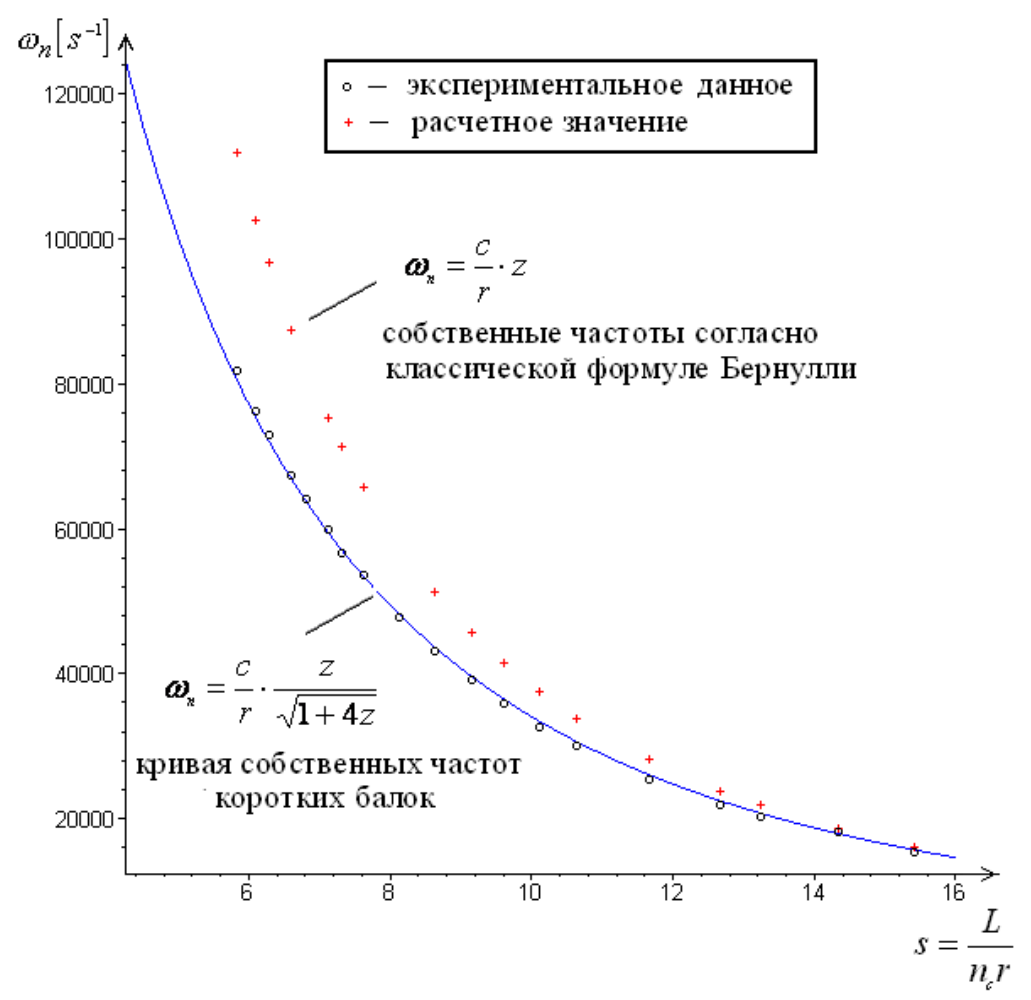

Рис.3. Кривая частот коротких балок в сравнении с их классическими значениями, без эффектов поворота и сдвига

Относительная ошибка между экспериментальными данными и аппроксимационной зависимостью (5) не превышает 5 \%. Можно отметить явное совпадение экспериментальных и аналитических результатов.

Чтобы получить универсальную зависимость для поперечных колебаний всех коротких балок, обратимся к дифференциальному уравнению свободных колебаний коротких балок, полученному С.П. Тимошенко [1]:

Chirikov V. A. et al. / Universal experimental relation for natural friquencies of transversal vibration of stubby free-free beams 


$$
\rho A \frac{\partial^{2} y}{\partial t^{2}}-\rho I\left(1+\kappa \frac{E}{G}\right) \frac{\partial^{4} y}{\partial t^{2} \partial x^{2}}+E I \frac{\partial^{4} y}{\partial x^{4}}+\kappa \frac{\rho^{2} I}{G} \frac{\partial^{4} y}{\partial t^{4}}=0,
$$

где: $y$ - поперечное перемещение оси балки; $x$ - продольная координата вдоль оси балки; $t$ - параметр времени; $A$ - площадь поперечного сечения; $I=A r^{2}$ - инерционный момент сечения; $\rho$ - массовая плотность материала балки; $E-$ модуль упругости; $G=\frac{E}{2(1+v)}-$ модуль сдвига, $\kappa$ - коэффициент формы поперечного сечения; $v$ - коэффициент Пуассона.

При его выводе используется уравнение связи для общего угла сдвига $\beta$, введенное С.П. Тимошенко в динамику короткой балки:

$$
\beta=\frac{\partial y}{\partial x}-\psi=\kappa \frac{V}{G A}
$$

где $\psi$ - угол наклона касательной к кривой изгиба при пренебрежении сдвигом; $V$ перерезывающая сила.

Эта связь играет главную роль при конвенциональном выводе уравнения, так как на основе используемых при выводе уравнения (6) теорем механики без нее вообще невозможно получить уравнение колебаний с учетом сдвига, потому что действие внутренних сил в элементе балки в этих теоремах вообще не учитывается. Если отбросить последний член в уравнении (6) из-за его малости второго порядка [2], то уравнение приобретает вид:

$$
\rho A \frac{\partial^{2} y}{\partial t^{2}}-\rho I\left(1+\kappa \frac{E}{G}\right) \frac{\partial^{4} y}{\partial t^{2} \partial x^{2}}+E I \frac{\partial^{4} y}{\partial x^{4}}=0
$$

а получаемая из него собственная частота колебаний $\omega_{n}$ балки, после подстановки в него общего решения:

$$
y=C_{n} \cdot \sin \left(p_{n} x\right) \cdot \sin \left(\omega_{n} t\right)
$$

где: $p_{n}=\frac{n_{c} \pi}{l}$,

приобретает следующий вид:

$$
\omega_{n}=\frac{c}{r} \cdot \frac{z}{\sqrt{1+(1+2 \kappa(1+v)) z}}
$$

где $z$ представляет собой выражение в (3) и (1).

Подставляя в (10) значения $\kappa=1,2$ для прямоугольных поперечных сечений балок и $v=0,25$ для стали, как раз и получается установленная экспериментальная зависимость (5). Так что, полученная аналитическая зависимость (10) является в действительности более полной при определении частот поперечных колебаний коротких балок, включая балки со свободными концами. Она позволяет проводить быстрые расчеты собственных частот поперечных колебаний коротких балок, предотвращая сложные алгоритмы и трудоемкие расчеты.

\section{4. Дискуссия}

Решение (10) приближенного уравнения Тимошенко короткой балки (8), полученное в предыдущем разделе, оставляет ряд вопросов. Один из них связан с тем, что используемое выражение формы колебаний балок в (9) является неполным для балок со свободными 
концами. Легко проверить, пользуясь соответствующими краевыми условиями, что полное выражение формы в этом случае имеет вид:

$$
f=A_{n} \cdot \cos \left(p_{n} x\right)+\operatorname{sh}\left(p_{n} x\right)+A_{n} \cdot \operatorname{ch}\left(p_{n} x\right)+\sin \left(p_{n} x\right),
$$

где: $A_{n}=\frac{\operatorname{sh}\left(p_{n} l\right)-\sin \left(p_{n} l\right)}{\cos \left(p_{n} l\right)-\operatorname{ch}\left(p_{n} l\right)}$.

При получении зависимости (10) в разделе 3 использован лишь последний член в выражении (11). Можно показать, что использование первого члена или суммы первого с последним членом формы также приведет к полученному результату. Для второго и третьего членов, однако, приблизительное уравнение (8) не удовлетворяется, так что уравнение колебаний в этом случае является неполным, а в качестве компенсации не может быть использован отброшенный член полного уравнения (6). Тем не менее, ввиду хорошего совпадения получаемых экспериментальных результатов с аналитической зависимостью (10), а также на основе множества проведенных расчетов с использованием современных специализированных программ инженерного проектирования, можно предположить, что влияние второго и третьего членов в соотношении (11) пренебрежимо мало по сравнению с остальными членами.

При этом можно заметить, что для балок на шарнирных опорах по концам, очевидно, получается та же самая зависимость (10) для собственных частот поперечных колебаний, что и для балок со свободными концами, поскольку форма колебаний балок с шарнирными условиями на концах точно совпадает с формой, используемой в (9).

Дополнительный вопрос возникает в связи с распространением универсального уравнения Тимошенко (6) вообще на все короткие балки. Нужно отметить, что в современных компьютерных пакетах инженерных расчетов заложена именно такая гипотеза. В пользу этой гипотезы служит и тот факт, что при выводе уравнения Тимошенко (6) не используются никакие специфические краевые условия. Кроме того, формы колебаний балок с различными условиями на концах всегда включают член (9). Отсюда следует, что и решение (10) для балок со свободными концами можно рассматривать как универсальную зависимость, верную при любых краевых условиях.

Можно также добавить, что полученное решение (10) дифференциального уравнения динамики коротких балок (8) распространяется на все возможные частоты колебаний балок, а не только на фундаментальные. При этом расчет поперечных частот колебаний коротких балок на основе универсальной зависимости (10) с применением предложенных параметров $s \quad$ и $z$ делается единообразно для всех балок. Необходимо только подставлять соответствующие числа $n_{c}$, различающиеся по специфическим условиям на концах балки и участвующие в формировании значения параметра $s$ в (1). Очевидно, что для балок с шарнирными опорами на концах значения параметра $s$ будут большими, чем для тех же балок со свободыми концами. Поэтому значения собственных частот балок с шарнирными опорами будут ниже. То же верно и для всех балок с другими краевыми условиями, кроме балок с жестко закрепленными концами, для которых получаются идентичные собственные частоты. Проводя расчеты с использованием современных инженерных пакетов, можно даже установить некоторое уменьшение собственных частот для балок с жестко закрепленными концами по сравнению с теми же балками со свободными концами. Такой факт видится довольно странным ввиду увеличения прочности балки при жестком закреплении.

\section{5. Заключение}

Таким образом, удалось установить, что „балка Тимошенко” наверняка представляет собой короткую балку со свободными концами. Это подтверждается серией экспериментов по установлению собственных частот поперечных колебаний свободных балок с

Chirikov V. A. et al. / Universal experimental relation for natural friquencies of transversal vibration of stubby 
прямоугольным поперечным сечением, проведенных как в лаборатории Токийского университета (Япония), так и в лаборатории Технической механики при ТУ (Варна). Это подтверждается и теоретически, посредством подстановки соответствующей формы колебаний балок со свободными концами в приближенное уравнение С.П. Тимошенко (8), в результате чего получается более полная зависимость (10), включающая в себя и полученную ранее зависимость (5).

Уравнение (8) можно преобразовать и к другому виду, подставляя известное общее уравнение поперечных колебаний стержня [2]

$$
E I \frac{\partial^{4} y}{\partial x^{4}}=-\rho A \frac{\partial^{2} y}{\partial t^{2}}
$$

в последний член преобразованного после раскрытия скобки, уравнения (8):

$$
\rho A \frac{\partial^{2} y}{\partial t^{2}}-\rho I \frac{\partial^{4} y}{\partial t^{2} \partial x^{2}}+E I \frac{\partial^{4} y}{\partial x^{4}}-\kappa \rho \frac{E I}{G} \frac{\partial^{2}}{\partial x^{2}}\left(\frac{\partial^{2} y}{\partial t^{2}}\right)=0 .
$$

Проводя очевидные упрощения по подстановке (12) в (13), получаем:

$$
\rho A \frac{\partial^{2} y}{\partial t^{2}}-\rho I \frac{\partial^{4} y}{\partial t^{2} \partial x^{2}}+E I \frac{\partial^{4} y}{\partial x^{4}}+\kappa \frac{(E I)^{2}}{G A} \frac{\partial^{6} y}{\partial x^{6}}=0 .
$$

В [5] показано, что точно такое же уравнение, но со знаком минус в последнем члене, является уравнением свободных поперечных колебаний короткой балки с шарнирно опертыми концами, а именно:

$$
\rho A \frac{\partial^{2} y}{\partial t^{2}}-\rho I \frac{\partial^{4} y}{\partial t^{2} \partial x^{2}}+E I \frac{\partial^{4} y}{\partial x^{4}}-\kappa \frac{(E I)^{2}}{G A} \frac{\partial^{6} y}{\partial x^{6}}=0 .
$$

При выводе этого уравнения уравнение связи (7) для общего угла сдвига $\beta$ не используется, так как применяется теорема изменения кинетической энергии, которая автоматически учитывает работу внутренних сил в элементе балки, порожденную сдвигом, без необходимости применения дополнительного уравнения для общего угла сдвига.

Решением частотного уравнения в этом случае будет

$$
\omega_{n}=\frac{c}{r} \cdot z \sqrt{\frac{1+2 \kappa(1+v) z}{1+z}},
$$

что очевидно имеет более высокие значения, чем (10) при одном и том же значении параметра $s$ и соответственно $z$.

Кроме того, проведенный в [3] анализ собственных частот поперечных колебаний балок с другими краевыми условиями (без использования уравнения для общего угла сдвига) показал, что кривая собственных частот коротких балок со свободными концами в действительности проходит ниже всех кривых коротких балок с другими краевыми условиями, а кривая балки с жестко закрепленными концами проходит выше всех.

Ввиду возникших несоответствий необходимо продолжать эксперименты по выявлению достоверных результатов. Поэтому представляется интересным на следующем этапе исследований провести эксперименты с короткими балками на шарнирных опорах по концам. Тем более, что почти все предпосылки для этого имеются. Необходимо только осуществить обеспечение на практике реальных шарнирных опор для короткой балки. Чисто проведенные эксперименты помогут решить возникшие вопросы, связанные с реальной картиной колебаний коротких балок и использовать полученные результаты для практических целей. 
open-access journal

\section{Благодарность}

Настоящая работа выполнена при частичной поддержке Национального фонда научных исследований Болгарии, ТУ-Варна, проект НП-7/2012 г.

\section{Литература}

1. Timoshenko S. P. On the Correction for Shear of the Differential Equation for Transverse Vibration of Prismatic Bars // Philosophical Magazine. - 1921. - Vol. 6, № 41. - P. 744-746.

2. Тимошенко С. П. Колебания в инженерном деле. - М : Наука, 1967. - 444 с.

3. Chirikov V. A. On the Range of Rotary Inertia and Shear Effects for Beams // Applied Mechanics in the Americas, PACAM VII, Temuco, Chile, 2-4 January, 2002. - 2002. - Vol. 9. P. 9-12.

4. Chirikov Victor A., Koichi Ozaki. Experimental Formula for Transverse Vibrations of Stubby Free-Free Beams // International Conference "Tehnonav 2004", Constantsa, Romania, 2004. 2004. - P. 25-28. - ISSN 1223-7221.

5. Чириков В. А. Уравнения поперечных колебаний коротких балок. - Деп. в ВИНИТИ 06.12.2005, № 1595-B2005.

6. Димитров Д. М. Използване на динамични методи за определяне на еластичните константи на материали получени по метода на праховата металургия // Научни известия на HTCM. - Юни 2013. - Година XXI, брой 2(139). - С.433-435. - ISSN 1310-3946.

7. Костов К. Определяне на собствени честоти на греди с разпределени параметри при свободно трептене // Юбилейна студентска научна сесия на ТУ-Варна, 04.04.2012.

8. ASTM E1876-09. Standard Test Method for Dynamic Young's Modulus, Shear Modulus, and Poisson's Ratio by Impulse Excitation of Vibration. - Book of Standards Volume : 03.01. - ASTM, USA, 2012. 